\title{
USER-CENTRIC PROCESS OF DESIGNING A MOLECULAR \& CELLULAR QUERY INTERFACE FOR BIOMEDICAL RESEARCH
}

\author{
T.S. Liaw ${ }^{1}$ and N. Gehlenborg ${ }^{1}$ \\ ${ }^{1}$ Harvard Medical School, United States of America \\ $\triangle$ nils@hms.harvard.edu
}

\begin{abstract}
There is a growing demand for the adoption of user-centric design processes for the development of computational biology software as usability becomes a major concern. Our team develops interfaces for a human biomolecular data portal with user-centered design, so we present a case study of the design process of a molecular and cellular query to emphasize the importance of user-centric design and reveal the complications that arise in a complex software development environment. The study follows the design process from user requirement gathering to the prototyping of a minimum viable product.
\end{abstract}

Keywords: life sciences and design, case study, design practices

\section{Introduction}

Although user-centric design processes have been well-developed over the years (Dopp et al., 2019; Ritter et al., 2014), complex domains, especially those impacted by the increasing role of computing such as artificial intelligence, lack a focus on usability practices and tend to not invest in user-centric development models (Chilana et al., 2010; Pavelin et al., 2012). However, there is a growing need for computational biology software developers to adopt a user-centered design philosophy for the development of their software interfaces since usability is increasingly becoming an essential factor for users to navigate the complexity of human biological data (List et al., 2017; Pavelin et al., 2012). With computational biology involving vast amount of complex data and computing algorithms, software products and tools from this field have consistently experienced similar usability issues such as convoluted user navigation, confusing biological terminology, software instability and installation difficulty (Bolchini et al., 2009; Mangul et al., 2019).

User-centered design (UCD) is an iterative design process that involves users to guide and inform the design and development process in order to gain an understanding of the requirements for a product or tool relevant to user needs (Ritter et al., 2014; Wallach and Scholz, 2012). Applying UCD for developing computational biology software, developers can improve user experience, and prevent the usability issues typically found in these types of interfaces (Macaulay et al., 2009; Pavelin et al, 2012; Walden et al., 2020). UCD can also significantly increase user adoption and satisfaction with the product, and reduce the cost and time of the software development (Bolchini et al., 2009; Mangul et al., 2019; Walden et al., 2020).

\subsection{The Human Biomolecular Atlas Program Data Portal}

The NIH-sponsored Human BioMolecular Atlas Program (HuBMAP) develops technologies for mapping the human body at a cellular resolution and generating spatial maps for tissues obtained from healthy individuals (HuBMAP Consortium, 2019). As part of the HuBMAP Integration, Visualization 
and Engagement (HIVE) center, our group focuses on developing the user interfaces for the HuBMAP Data Portal (portal.hubmapconsortium.org), which allows user exploration of the consortium's data for its diverse anatomical, histological, cellular, molecular and genomic metadata (HuBMAP Consortium, 2019). The data portal focuses on users who intend to browse, query and download relevant data in order to further their scientific discovery within their specific biomedical field. Our group applies UCD for the design and implementation of these features in order to ensure the data portal satisfies user requirements.

\subsection{Molecular and Cellular Query Interface}

Within the human-computer interaction field, design guidelines that support the development of userfriendly search interfaces exist to allow users to easily formulate their queries, understand their search results and track their information gathering process (Hearst, M., 2009). Experimental and computational biologists rely on these types of digital search interfaces to seek out information relevant for their respective biomedical research fields, primarily databases for biomedical literature such as PubMed (Iñiguez-Jarrin et al., 2019). Given a lack of user research practices within the computational biology field, as previously discussed, design guidelines and practices for developing in-depth biomedical informational query interfaces have not been well established.

The HuBMAP Data Portal currently supports a faceted search feature in which users can browse through donor, sample or dataset data. By selecting any individual data, a user can interact with its visualization tools, view the data provenance, or download the relevant metadata. Our team designed this feature by gathering user feedback from prior workshops and surveying similar data portals supporting faceted search interfaces.

However, this feature does not support any queries into the molecular or cellular data available within the HuBMAP Data Portal and provide limited support for the analysis of the data. Our team proposed to conduct user research in order to properly design a query interface that would allow access and exploration of the molecular and cellular metadata. With prior user research and persona development, we focused our user research on an interview study since interviews can provide in-depth information about the priorities of users (Dopp et al., 2019; Lack, 2007).

The paper emphasizes the importance of employing a user-centered design process for designing biomedical computational interfaces and the complications of implementing the design results in a complex software development environment by discussing a case study of our process when designing and developing a biologically-based query interface for the HuBMAP Data Portal. This discussion includes the user research for gathering user requirements (Section 2) and its results (Section 3). We present the scope of a minimum viable product (MVP) given limitations of the supporting API, a flow diagram summarizing user navigation, and interactive prototypes, which were handed off to the user interface (UI) development team (Section 4). The implementation process of the proposed design will be discussed with a focus on the difficulties caused by the separation of development responsibilities given the complexity of the feature, with the supporting API developed by a collaborating software development team (Section 5).

\section{User Research}

\subsection{Preliminary Understanding of User Requirements}

\subsubsection{Personas \& Prior User Research}

Personas, representations of the targeted users' characteristics and their values (Cooper 1999), for the usages of HuBMAP tools were created prior to the development of the data portal with user groups including: data generators, data analysts, technologists and educators/learners. As the molecular and cellular query targets users who intend to browse and analyse the data, we determined that the main users to target for feedback were experimental and computational biologists who intend to interact with the biological data. Personas related to the target user group helped determine the recruitment of participants for user research. 
Previous workshops were conducted to gather informal feedback from users interested in interacting with HuBMAP data. This information formed the basis for preliminary use cases, which were used during user interviews to narrow the scope of the feedback to be within the realm of the upcoming feature. These use cases were formulated in terms of potential research questions, and users were asked to expand on whether or not these research questions were pertinent to their current work. The research questions were:

a) How homogeneous is a tissue? (e.g. what fraction of the tissue is of the most common cell type or distribution of cell types?)

b) How abundant are $\mathrm{X}$ cell type in $\mathrm{Y}$ organ?

c) What is the expression of gene/protein in X cell type in $\mathrm{Y}$ organ?

We conducted a literature review and an informal evaluation of other biological query tools in order to gather typical user interface features and user task flows. Given the limited amount of existing biological query interfaces and the possibility of these interfaces being not supported by user research, other query interfaces were also evaluated, especially queries involving large amounts of complex data and query interfaces from sources known to use UCD as a driving design process supporting their interfaces.

\subsubsection{API Requirements}

The API supporting the query feature was being developed simultaneously to the design process by a separate software development team within the HIVE. In order to better understand the API that supports the interface, our development team communicated with the API development team and created a Python client accessing the API. We found that the API development team was planning to support six types of queries, prior to the completion of the user research for the molecular and cellular query (Table 1). We planned to validate the user requirement for the six queries during the user research process to justify the development direction of the API, and gather user feedback regarding the planned queries to improve the usability of the queries to better fit specific user needs.

Table 1. Planned Queries by the API

\begin{tabular}{|l|l|l|}
\hline Input & Output & Description \\
\hline Gene Expression & Cell & $\begin{array}{l}\text { Retrieve cells with expression of gene X above a } \\
\text { significance level of Y. }\end{array}$ \\
\hline Gene Expression & Organ & $\begin{array}{l}\text { Retrieve organs associated with expression of gene X } \\
\text { above a significance level of Y. }\end{array}$ \\
\hline Protein Expression & Cell & $\begin{array}{l}\text { Retrieve cells with expression of protein X above a } \\
\text { significance level of Y. }\end{array}$ \\
\hline Cell & Organ & Retrieve organs represented in selected set of cells. \\
\hline Organ & Gene Name & $\begin{array}{l}\text { Retrieve genes expressed at significant level Y within } \\
\text { selected organ(s). }\end{array}$ \\
\hline Organ & Cell & Retrieve set of cells represented in selected organ. \\
\hline
\end{tabular}

\subsection{User Interviews}

We conducted 17 semi-structured interviews with participants recruited based on the identified target user group of experimental or computational biologists for the upcoming query feature. The majority of the individuals were involved in the biological data contribution process of HuBMAP as part of the Tissue Mapping Centers (TMCs) as either principal investigators, postdoctoral researchers or research staff. Participants were asked to self-identify as experimental biologists $(\mathrm{N}=7)$, computational biologists $(\mathrm{N}=4)$ or both $(\mathrm{N}=6)$.

Interview questions were formulated around the understanding and gathering of user feedback related to: (1) user's general usage and requirements of the HuBMAP Data Portal, (2) importance of the planned queries supported by the API to the user's work, (3) additional query proposals useful to the user's work and (4) desired outputs of the query results. 
Interviews were conducted remotely through video call individually for a duration of 20-30 minutes each. Recordings were made with user permission to accurately transcribe the interview and deleted once transcripts were verified.

\section{Results}

\subsection{Interview Analysis}

nformation was extracted from the qualitative interview transcripts with interview coding techniques (Weston et al., 2001) and organized into meaningful categories for analysis with affinity mapping. Affinity mapping is a user research technique used to consolidate and structure information to highlight significant insights (Wallach and Scholz, 2012). We used affinity mapping to analyse the large amount of qualitative data from the user interviews. Figure 1 shows a portion of the affinity diagram for the user interviews of the category containing the user-desired output type of a visualization.

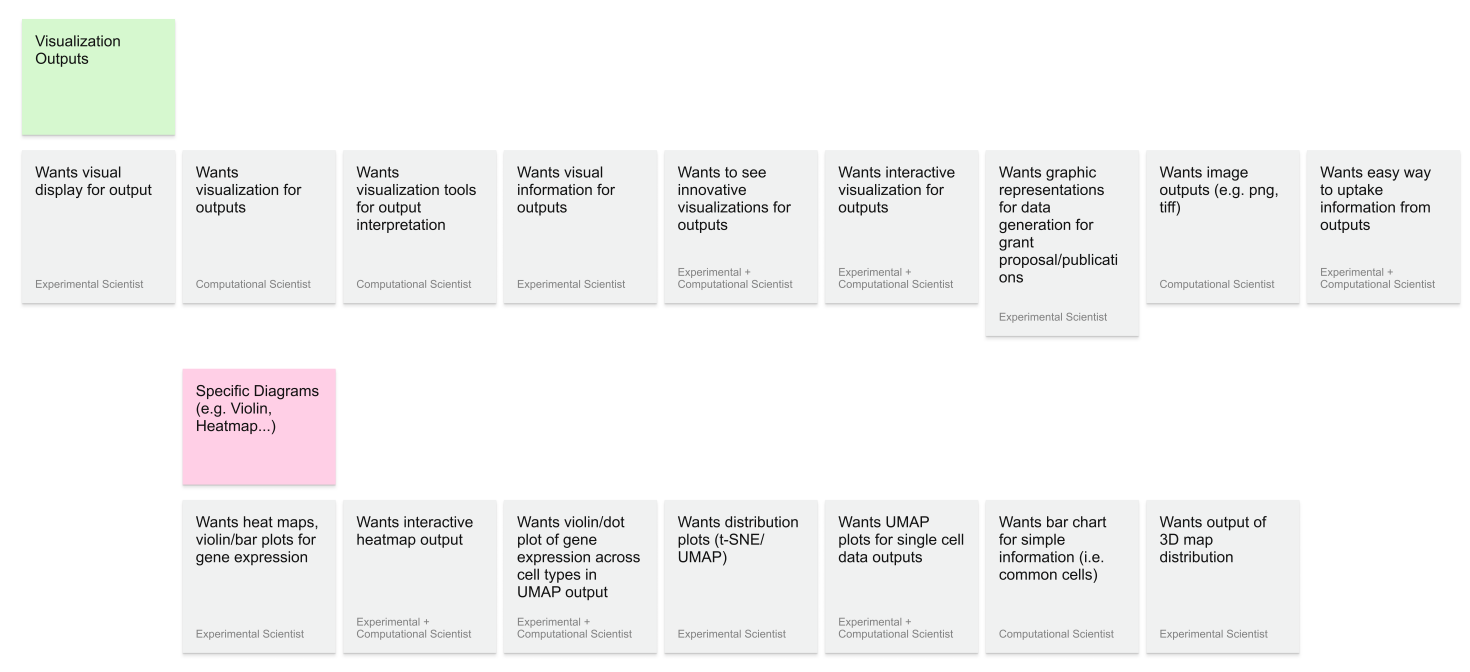

Figure 1. Affinity Diagram Category: User-Desired Output

\subsection{Planned API Queries}

To validate the ongoing API development direction, users were presented with a list of planned API queries from Table 1 during the interview and asked to identify and explain whether or not that query would be useful for their work. For all queries, the majority of users generally agreed that the queries would be useful. For some queries, users indicated certain criteria that would improve the query's usefulness. For one of these queries, gene expression to organ $(\mathrm{N}$ useful $=6)$, users mentioned they would prefer a more specific output other than organ, such as tissue or specimen type. These user comments were useful in validating the queries and improving the planned queries' biological terminology to be more user-friendly. Table 2 shows the results of users' opinions on the planned queries. Not all users commented on every query, and those users were not counted in any fields.

Table 2. User Interview Results for Planned Queries

\begin{tabular}{|c|c|c|c|l|}
\hline Query Type & Useful & Might be Useful & Not Useful & \multicolumn{1}{|c|}{ General User Comments } \\
\hline Gene Expression $\rightarrow$ Cell & 8 & 3 & 1 & Requires additional context. \\
\hline Gene Expression $\rightarrow$ Organ & 6 & 4 & 2 & Output should be more specific. \\
\hline Protein Expression $\rightarrow$ Cell & 8 & 2 & 1 & Requires additional context. \\
\hline Cell $\rightarrow$ Organ & 7 & 0 & 4 & - \\
\hline Organ $\rightarrow$ Gene Name & 5 & 3 & 2 & $\begin{array}{l}\text { Wants ability to adjust cellular } \\
\text { composition }\end{array}$ \\
\hline Organ $\rightarrow$ Cell & 10 & 3 & 0 & Requires additional context \\
\hline
\end{tabular}




\subsection{User Proposed Queries}

Users were asked to propose any queries, outside of the planned API queries, that would be beneficial to their work. The most mentioned queries were cell type queries $(\mathrm{N}=11)$ and spatial queries $(\mathrm{N}=8)$; users mentioning spatial queries specified queries focused on tissue localization, cell subsets within structural imaging data, spatial information of cell type/expression level or cell distribution. Users also wanted queries supporting certain biomolecules $(\mathrm{N}=7)$, including metabolites $(\mathrm{N}=3)$, lipids $(\mathrm{N}=3)$, proteins $(\mathrm{N}=3)$ and multi-omics data $(\mathrm{N}=2)$.

Other queries worth mentioning from the user interviews include donor information queries $(\mathrm{N}=5)$, cell interaction queries $(\mathrm{N}=3)$, functional tissue unit queries $(\mathrm{N}=3)$, gene regulation queries $(\mathrm{N}=2)$ and longitudinal studies queries $(\mathrm{N}=2)$.

Most of these queries cannot be currently supported in the data portal, but many of these user requirements will eventually be addressed due to ongoing work by other teams within the consortium to provide the appropriate technologies.

\subsection{Query Outputs}

The most desired output users mentioned were visualizations $(\mathrm{N}=11)$. Specific visualization types mentioned were heat maps, bar plots, UMAP/t-SNE plots, dot plots, violin plots and 3D map distributions. Users also mentioned wanting additional context and external links provided with their outputs in order to better understand their results $(\mathrm{N}=9)$. Specifics of these additional context mentioned were abbreviation definitions, cell type or cell region contexts, cell cluster definitions, related metadata and external image links. Provenance related to the output was also commonly mentioned by users $(\mathrm{N}=6)$, as well as the inclusion of the output's raw data in either a downloadable format or within a spreadsheet on the interface $(\mathrm{N}=6)$.

Other output types worth mentioning include a list-based output $(\mathrm{N}=4)$, ability to self-annotate $(\mathrm{N}=3)$ and inclusion of a quality control or confidence score with their outputs $(\mathrm{N}=2)$.

\subsection{User Research Findings Report}

The results from the user research was reported to internal stakeholders within the consortium. Documentation of general use cases for the data portal was updated with the user interview responses of the biologists' general usage of the data portal for their specific research purposes.

Although some user-proposed queries require technologies still in development, the report delivered the scope of the user requirements that can help determine the future direction of the work related to the molecular and cellular query. This information can serve as a base in the future for the teams developing the supporting technologies to launch further user research on those topics.

\section{User Flow \& User Interface Designs}

\subsection{API Limitations}

Monthly meetings with the API development team occurred throughout the design and implementation process so that the API could meet the desired minimal requirements by the time of implementation. Throughout the user research process, the UI development team was exploring the API through a Python client in order to understand the constantly evolving state, ensure the API was aligned with the ongoing results from the user research and communicate any major issues found to the API development team to resolve before UI implementation. After gathering user requirements, we found that the current state of the API would not be able to return all of the planned queries as previously expected and had significantly slow API response. Therefore, we proposed creating a minimum viable product (MVP) that would satisfy the minimum user requirements within the limits of the current state of the API, and consider the slow API responses when designing any interfaces to prevent user confusion.

\subsection{Minimum Viable Product}


Given the API limitations, two user requirements, formulated in terms of query types, were focused on for the MVP of the molecular and cellular query interface. These user requirements were:

1. From datasets selected from the faceted search interface, return datasets that contain at least one cell where a user-selected gene or protein $\mathrm{X}$ is expressed above a threshold value $\mathrm{Y}$.

2. For a given dataset, show visualization(s) where the cells that express gene/protein $\mathrm{X}$ above a threshold value Y.

The first user requirement reflected the most basic case, as gathered from the user research, of exploration of the genomic or proteomic cellular data within the datasets. For this scenario, we intended to return every dataset matching the query parameters from the list of user-selected datasets.

The second user scenario reflected user feedback regarding the user-desired output types of a visualization corresponding to their results. Implementation of the second user requirement was to be built off of the first scenario results as we planned to generate visualizations for every dataset from the resulting list of datasets that matched the query parameters from the first scenario. We determined that two visualizations would sufficiently fulfil the second requirement: a bar chart showing the cells expression level of the selected gene or protein above a certain threshold value, and a bar chart showing the distribution of cells across cell clusters (Figure 3d). These visualizations would also indicate the percentages of cells matching in total within the datasets as well as the percentage of cells matching in cell clusters.

\subsection{Flow Diagram}

User flow diagrams are typically used as an overview of user navigation through a product and help draft the path users undertake when interacting with the system in order to predict potential user actions and its consequences (Dopp et al., 2019). Therefore, we created a flow diagram for the MVP (Figure 1), detailing the user navigation through the two user scenarios, and annotated the diagram for the interfaces and interactions that would be required to be built to support the user flow. This diagram was used for communication of the design between the API and the UI development team, and iterated upon given feedback on any technological limitations or design-related issues. Initial wireframes of the potential UI were created after initial validation of the user flow diagram to help with communication visually for additional feedback. The user flow diagram and the basic wireframes led to the final design step of creating a high-fidelity interactive prototype. Figure 2 shows a simplified version of the flow diagram without annotations and wireframes.

The user flow diagram helped with certain design decisions including whether or not the molecular and cellular query should be a new feature on the portal or built off an existing feature. Since the MVP requires an input of a list of datasets, we determined to integrate the new feature with the existing faceted search interface since users could select and facet on a list of datasets before narrowing their query for the molecular and cellular information of those datasets.

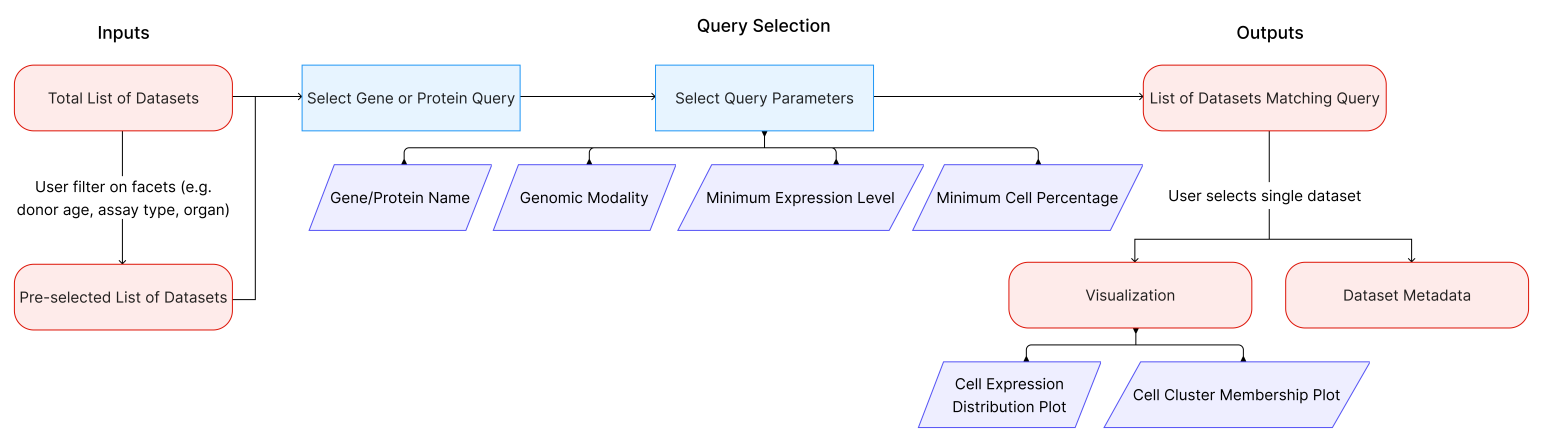

Figure 2. User Flow Diagram

\subsection{Query Interface Design}

Interactive prototypes and still-frame mock-ups were created with Figma, a web-based prototyping tool (Figma, 2016). Prototypes went through several iterations given feedback from the API and UI 
development team. Formal user testing of the prototypes prior to implementation was not conducted, but feedback from consortium members was collected whenever prototypes were shared during internal meetings.

\subsubsection{User Navigation}

Figure 2 shows mock-ups of the query interface, which were slightly modified to fit the conforms of the paper, and the user navigation steps through the query from dataset(s) selection launched from the existing faceted search interface (Figure 3a) to the user sending the list of datasets to the advanced query selection interface. To narrow the initial list of datasets to find relevant molecular and cellular information, the user can select a gene or protein query from the query selection interface (Figure $3 b$ ). The user can then select parameters of their chosen query, with Figure 3c showing the parameter selection of a gene query. After launching the query, the users receive their results of a list of datasets that matches the query parameters set by the user, in the form of a table with metadata as columns. Each dataset should have two graphical representations, in the form of bar charts, of the dataset's cell and cell cluster information matching query parameters (Figure 3d).

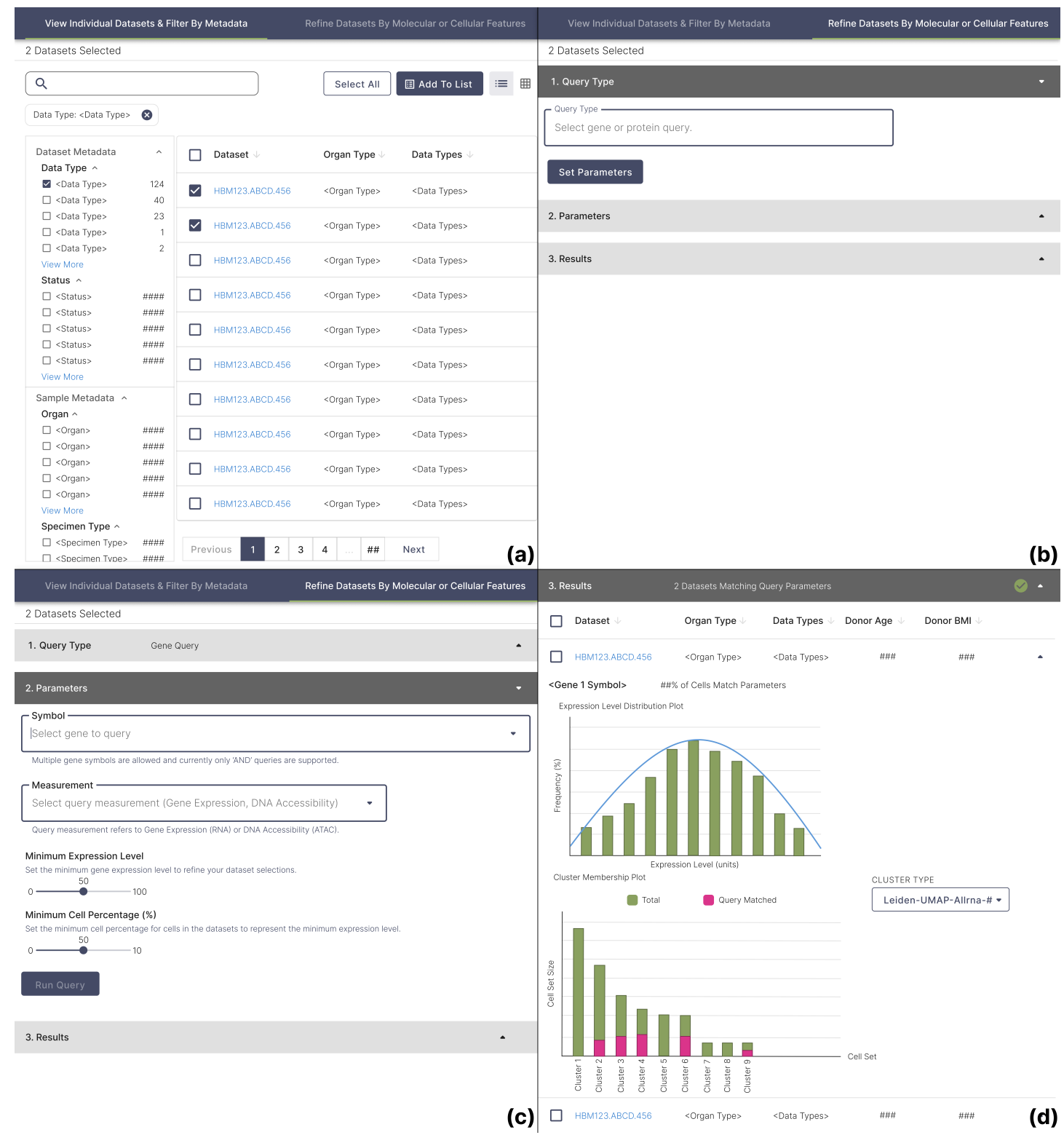

Figure 3. (a) Dataset(s) Selection from Faceted Search (b) Gene or Protein Query Selection (c) Query Parameters Selection (d) Query Results 


\section{Implementation}

\subsection{API Limitations}

The API by the time of design handoff to the UI development team had several issues that persisted throughout the implementation process. These issues included: (1) simultaneous development of the API during the UI development, (2) API unstableness reflected by how a few requests call by a single user can lead to outages and (3) an incomplete API as some features expected to be returned by the API did not work as intended. These issues discovered during the implementation process were conveyed to the API development team.

\subsection{Implementation Results}

Complete implementation of the query user interface was severely impacted by the limitations of the API. User flow of the query and all visual features of the planned design are currently implemented, but the API limitations prevents any useful user interaction unless the user understands the exact query parameters that will work with the API. Given the unstableness and slow response of the API, users could potentially be querying a supported request, but the API might not return the result as intended within a reasonable timeframe.

Therefore, the link to the molecular and cellular query is not currently referred to on the portal and can only be accessed if the link is known to the user. Although the original design of the new query feature was to be an addendum to the faceted search interface, the molecular and cellular query was not integrated in order to not impact the current user experience. The existing UI for this new feature is currently useful only for minimal user testing to verify the UI design or as a reference for internal stakeholders. In order to complete implementation, the API development team will have to solve the issues previously mentioned.

\section{Discussion of the API}

The situation of the API complications affecting implementation completion of the proposed design reveals the complexity that can exist in a field such as computational biology. The current organization of our software development teams and the separation of development responsibilities between the different teams resulted as a consequence of the difficulties of implementing the technologies required to support a feature such as the molecular and cellular query. Complications can arise from this type of organization, as seen throughout the design and implementation process, from the teams perhaps having different priorities and timelines for development, or when user research is conducted in parallel to portions of the development process. By conducting user research, however, we were able to detect the usability issues before users were able to interact with the actual feature. Usability issues that were caught prior to implementation included software instability and confusing biological terminology, which are common usability issues in computational biology software that can lead to user rejection of a feature. By understanding the usability issues, the API development team can understand more specifically on which aspects of the API to fix and improve on. For those involved in the UI design and development process, any design work can take into account any usability issues that will continue to persist, even with fixes to the API.

\section{Conclusion}

The design and development process of a biological query interface was explored throughout this paper to highlight the importance of employing a user-centric design philosophy in the computational biology field. By conducting user research when developing an interface that is reliant on complex technologies, we were able to better understand the users of the data portal and gain an in-depth understanding of their specific needs for the exploration and analysis of biological data on a molecular and cellular level. With a lack of a standardized design guidelines or a standard for creating a complex biological query interface, the findings of this research can also encourage others creating similar interfaces to conduct their own 
user research in order to better design their query interfaces into human biological information databases.

As many aspects of the results from the user research were not addressed in the final design of the query interface due to API limitations and the lack of technologies to support some of the user requests because of ongoing work, the user requirements, such as spatial or cell type queries, or desired output types, such as raw data downloads or provenance, can help guide the direction of the future work on the molecular and cellular query as more advanced features become supported.

\subsection{Future Work}

The molecular and cellular query user interface will continue development once the API development team improves the API to reach production quality stability to allow implementation of the MVP to be finished. Once implementation is complete, user testing of the feature will allow us to understand more about the user requirements for these types of biological queries, and allow us to reiterate on the interface design to encourage user adoption of the feature. The molecular and cellular query will continue to be improved upon by adding queries based on the user proposals from the user research results and integrating additional user-desired output types. The user research from this study will guide the design process for those additional features.

\section{Acknowledgements}

This research is supported by the NIH Common Fund (NIH/OD OT2OD026677). The views expressed are those of the authors and not necessarily the NIH. The authors would like to acknowledge Chuck McCallum and John Conroy for leading the UI implementation, Margaret Vella for assistance throughout the user interview process, and all HuBMAP Consortium members who participated in the interviews.

\section{References}

Bolchini, D., Finkelstein, A., Perrone, V. and Nagl, S. (2009), "Better bioinformatics through usability analysis", Bioinformatics, Vol. 25 No. 3, pp. 406-412. https://doi.org/10.1093/bioinformatics/btn633

Chilana, P.K., Wobbrock, J.O. and Ko, A.J. (2010), "Understanding Usability Practices in Complex Domains", In Proceedings of the SIGCHI Conference on Human Factors in Computing System (CHI '10), Association for Computing Machinery, New York, pp. 2337-2346. https://doi.org/10.1145/1753326.1753678

Cooper A. (1999), "The Inmates are Running the Asylum", In: Arend, U., Eberleh, E. and Pitschke, K. (Eds.), Software Ergonomie '99. Berichte des German Chapter of the ACM, Vieweg+Teubner Verlag, Wiesbaden. https://doi.org./10.1007/978-3-322-99786-9 1

Dopp, A., Parisi, K., Munson, S. and Lyon, A. (2019), "A glossary of user-centered design strategies for implementation experts", Translational Behavioral Medicine, Vol. 9 No. 6, pp. 1057-1064. https://doi.org/10.1093/tbm/iby119

Figma. (2016). Figma, Inc.

Hearst, M. A. (2009), Search User Interfaces, Cambridge University Press, New York. https://doi.org/10.1017/CBO9781139644082

HuBMAP Consortium. (2019), "The human body at cellular resolution: the NIH Human Biomolecular Atlas Program", Nature, Vol. 574 No. 7777, pp. 187-192. https://doi.org/10.1038/s41586-019-1629-x

Iñiguez-Jarrin, C., Panach, J.I. and Pastor, O. (2019), "User-Centered Design for Biomedical Search User Interfaces", In: Andersson, B., Johansson, B., Barry, C., Lang, M., Linger, H., et al. (Eds.), Advances in Information Systems Development. Springer, Lund, Sweden, pp. 247-264. https://doi.org/10.1007/978-3-03022993-1 14

Lack, R. (2007), “The Importance of User-Centered Design: Exploring Findings and Methods", Journal of Archival Organization, Vol. 4 No. 1-2, pp. 69-86. https:doi.org/10.1300/J201v04n01 05

List, M., Ebert, P. and Albrecht, F. (2017), "Ten Simple Rules for Developing Usable Software in Computational Biology”, PLoS Computational Biology, Vol. 13 No. 1. https://doi.org/10.1371/journal.pcbi.1005265

Macaulay, C., Sloan, D., Jiang, X., Forbes, P., Loynton, S., et al. (2009), "Usability and User-Centered Design in Scientific Software Development", IEEE Software, Vol. 26 No. 1, pp. 96-102. https://doi.org/10.1109/MS.2009.27

Mangul, S., Martin, L.S., Eskin, E. and Blekhman, R. (2019), "Improving the usability and archival stability of bioinformatics software", Genome Biology, Vol. 20 No. 47. https://doi.org/10.1186/s13059-019-1649-8 
Pavelin, K., Cham J.A., de Matos, P., Brooksbank, C., Cameron, G., et al. (2012), "Bioinformatics Meets UserCentred Design: A Perspective", PLoS Computational Biology, Vol 8. No. 7. https://doi.org/10.1371/journal.pcbi.1002554

Ritter, F.E., Baxter, G.D. and Churchill, E.F. (2014), "User-Centered Systems Design: A Brief History”, In: Foundations for Designing User-Centered Systems, Springer, London, pp. 33-54. https://doi.org/10.1007/978-1-4471-5134-0 2

Walden, A., Garvin, L., Smerek, M. and Johnson, C. (2020), "User-centered design principles in the development of clinical research tools", Clinical Trials, Vol. 17 No. 6, pp. 703-711. https://doi.org/10.1177/1740774520946314

Wallach, D. and Scholz, S.C. (2012), "User-Centered Design: Why and How to Put Users First in Software Development", In: Maedche, A., Botzenhardt, A. and Neer, L. (Eds.), Management for Professionals, Springer, Berlin, pp. 11-38. https://doi.org/10.1007/978-3-642-31371-4 2

Weston, C., Gandell, T., Beauchamp, J., McAlpine, L., Wiseman, C. and Beauchamp, C. (2001). “Analyzing Interview Data: The Development and Evolution of a Coding System”, Qualitative Sociology, Vol. 24, pp. 381-400. https://doi.org/10.1023/A:1010690908200 